ARTICLE

\title{
Oxygen vacancy-driven orbital multichannel Kondo effect in Dirac nodal line metals $\mathrm{IrO}_{2}$ and $\mathrm{RuO}_{2}$
}

\author{
Sheng-Shiuan Yeh ${ }^{1,2,3}$, Ta-Kang Su${ }^{1}$, An-Shao Lien ${ }^{1}$, Farzaneh Zamani ${ }^{4}$, Johann Kroha (1) ${ }^{4}$, Chao-Ching Liao ${ }^{1}$, \\ Stefan Kirchner (iD) ${ }^{5,6 凶}$ \& Juhn-Jong Lin (i) ${ }^{1,2,7 凶}$
}

Strong electron correlations have long been recognized as driving the emergence of novel phases of matter. A well recognized example is high-temperature superconductivity which cannot be understood in terms of the standard weak-coupling theory. The exotic properties that accompany the formation of the two-channel Kondo (2CK) effect, including the emergence of an unconventional metallic state in the low-energy limit, also originate from strong electron interactions. Despite its paradigmatic role for the formation of non-standard metal behavior, the stringent conditions required for its emergence have made the observation of the nonmagnetic, orbital 2CK effect in real quantum materials difficult, if not impossible. We report the observation of orbital one- and two-channel Kondo physics in the symmetryenforced Dirac nodal line (DNL) metals $\mathrm{IrO}_{2}$ and $\mathrm{RuO}_{2}$ nanowires and show that the symmetries that enforce the existence of DNLs also promote the formation of nonmagnetic Kondo correlations. Rutile oxide nanostructures thus form a versatile quantum matter platform to engineer and explore intrinsic, interacting topological states of matter.

\footnotetext{
${ }^{1}$ NCTU-RIKEN Joint Research Laboratory, Institute of Physics, National Chiao Tung University, Hsinchu 30010, Taiwan. ${ }^{2}$ Center for Emergent Functional Matter Science, National Chiao Tung University, Hsinchu 30010, Taiwan. ${ }^{3}$ International College of Semiconductor Technology, National Chiao Tung University, Hsinchu 30010, Taiwan. ${ }^{4}$ Physikalisches Institut and Bethe Center for Theoretical Physics, Universität Bonn, Nussallee 12 , D-53115 Bonn, Germany. ${ }^{5}$ Zhejiang Institute of Modern Physics and Department of Physics, Zhejiang University, Hangzhou 310027, China. ${ }^{6}$ Zhejiang Province Key Laboratory of Quantum Technology and Device, Zhejiang University, Hangzhou 310027, China. ${ }^{7}$ Department of Electrophysics, National Chiao Tung

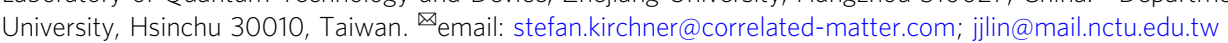


nconventional metallic states and the breakdown of the Landau Fermi liquid paradigm is a central topic in contemporary condensed matter science. A connection with high-temperature superconductivity is experimentally well established but the conditions under which these enigmatic metals form has remained perplexing ${ }^{1}$. One of the simplest routes to singular Fermi liquid behavior, at least conceptually, is through two-channel Kondo (2CK) physics ${ }^{2-4}$. Despite this long-standing interest, $2 \mathrm{CK}$ physics has thus far only been demonstrated to arise in carefully designed semiconductor nanodevices in narrow energy and temperature $(T)$ ranges $^{5-8}$, while claims of its observation in real quantum materials are contentious (see "Discussion" section for details). More recently, the interest in Dirac and Weyl fermions within a condensed matter framework has led to the exploration of the effects of strong spin-orbit coupling (SOC) and of topological states which are rooted in a combination of time-reversal, particle-hole, and space-group symmetries ${ }^{9,10}$. While there has been considerable progress in understanding weakly correlated topological metals, only a few materials have been identified as realizing topological phases driven by strong electron correlations, which includes the Weyl-Kondo semimetals $^{11}$. This raises the question if the $2 \mathrm{CK}$ counterpart of such a Weyl-Kondo semimetal, featuring an entangled ground state of the low-energy excitations of the $2 \mathrm{CK}$ effect with band-structure enforced Dirac or Weyl excitations, could at least in principle be stabilized. Exploring such a possibility, however, hinges on whether the 2CK effect can be stabilized at all in native quantum matter.

In this work we establish that oxygen vacancies ( $V_{\mathrm{O}}$ 's) in the Dirac nodal line (DNL) materials $\mathrm{IrO}_{2}$ and $\mathrm{RuO}_{2}$ drive an orbital Kondo effect. $V_{\mathrm{O}}$ 's are prevalent in transition-metal oxides, including, e.g., $\mathrm{TiO}_{2}$ and $\mathrm{SrTiO}_{3}$, and their properties and ramifications have become central research topics as they can lead to an intricate entanglement of spin, orbital, and charge degrees of freedom ${ }^{12-15}$. The active degree of freedom in the orbital Kondo effect is not a local spin moment but a 'pseudospin' formed by orbital degrees of freedom ${ }^{4}$. In $\mathrm{IrO}_{2}$ and $\mathrm{RuO}_{2}$, the orbital Kondo effect is symmetry stabilized by the space-group symmetries of the rutile structure (Fig. 1). Both materials have been characterized as topological metals which feature symmetry-protected DNLs in their Brillouin zones ${ }^{16,17}$. This provides a link between the formation of the orbital Kondo effect and the presence of DNLs. In $\mathrm{IrO}_{2}$ a nonmagnetic $2 \mathrm{CK}$ ground state ensues, while in $\mathrm{RuO}_{2}$ the absence of time-reversal symmetry results in an orbital one-channel Kondo (1CK) effect.

The rutile structure type possesses mirror reflection, inversion, and a fourfold rotation $\left(C_{4}\right)$ symmetry which enforce the presence of DNLs in the band structure of rutile oxides ${ }^{10}$. Some of these DNLs are protected from gapping out due to large SOC by the non-symmorphic symmetry of the rutile structure ${ }^{18,19}$. For $\mathrm{IrO}_{2}$ and $\mathrm{RuO}_{2}$ this has been recently confirmed by angleresolved photoemission spectroscopy and band structure studies $^{16,17,19}$. In the vicinity of $V_{\mathrm{O}}$ 's, this set of symmetries promotes the formation of the orbital $1 \mathrm{CK}$ and $2 \mathrm{CK}$ effect. The emergent Majorana zero mode that accompanies the formation of the $2 \mathrm{CK}$ effect is reflected in a singular excitation spectrum above the ground state which generates a $\sqrt{T}$-dependence of the resistivity $\rho(T)$ below a low- $T$ energy scale ${ }^{20}$, the Kondo temperature $T_{\mathrm{K}}$. This requires a well-balanced competition of two otherwise independent and degenerate screening channels and makes the 2CK effect extremely difficult to realize, especially in a natural quantum material ${ }^{4,21,22}$. If one channel dominates over the other, the low- $T$ behavior will be that of conventional fermions. If the $2 \mathrm{CK}$ state arises out of orbital Kondo scattering, magnetic-field $(B)$ independence is expected for field strengths well above $T_{\mathrm{K}}$ as long as $g \mu_{\mathrm{B}} B \ll W$, where $g$ is the Lande factor, $\mu_{\mathrm{B}}$ is the Bohr magneton, and $W$ is the conduction electron half-bandwidth. Our study is based on rutile $\left(\mathrm{MO}_{2}, M=\mathrm{Ir}, \mathrm{Ru}\right)$ nanowires (NWs) which allow us to combine a high degree of sample characterization with an exceptional measurement sensitivity while probing material properties in the regime where the characteristic sample dimension is much larger than the elastic electron mean free path (cf. Supplementary Note 3). That is, we are concerned with weakly disordered, diffusive metals which are threedimensional (3D) with respect to the Boltzmann transport, whereas strong correlation effect causes a resistivity anomaly at low $T$. Table 1 lists the relevant parameters for the NWs studied in this work.

\section{Results}

Oxygen vacancies in transition-metal rutiles $\mathbf{M O}_{2}$. In Fig. 1a, the vicinity of an $V_{\mathrm{O}}$, denoted $V_{\mathrm{O} 1}$, is shown. The metal ions surrounding $V_{\mathrm{O} 1}$, labeled $M 1, M 2$, and $M 3$, form an isosceles triangle (Fig. 1b). For the sites $M 1$ and $M 2$, an almost perfect $C_{4 v}$ symmetry exists which implies a corresponding degeneracy associated with the two-dimensional irreducible representation of $C_{4 v}$, see Fig. $1 \mathrm{c}$ and Supplementary Note 4 . In the pristine system, the metal ions are surrounded by oxygen octahedra anchored around the center and the corners of the tetragonal unit cell. The $\pi / 2$ angle between adjacent octahedra leads to a fourfold screw axis symmetry. This non-symmorphic symmetry not only protects DNLs in $\mathrm{IrO}_{2}$ against SOC-induced splitting ${ }^{17,19}$. It has also been linked to the high electrical conductivity of $\mathrm{IrO}_{2}$ (ref. ${ }^{10}$ ) and, as we find, is in line with the strong tendency to localize electrons near $V_{\mathrm{O}}$ 's required for the formation of orbital Kondo correlations. Moreover, the fourfold screw axis symmetry ensures that the $C_{4}$ rotation axes centered at the sites $M 1$ and $M 2$ near $V_{\mathrm{O} 1}$ are not parallel $\left(\hat{z}^{\prime} H \hat{z}\right.$, see Fig. $\left.1 \mathrm{~d}\right)$. This enhances the phase space for the orbital Kondo effect over orbital order linking sites M1 and M2 (see also Supplementary Note 5).

Experimental signatures of orbital 2CK effect in $\mathrm{IrO}_{2} \mathrm{NWs}$. Now we turn to our experimental results which, to the best of our knowledge, demonstrate the most convincing realization of the long searched orbital $2 \mathrm{CK}$ effect in a solid. Fig. 2 demonstrates the formation of an orbital $2 \mathrm{CK}$ effect in $\mathrm{IrO}_{2} \mathrm{NWs}$. We find that as $T$ decreases from room temperature to approximately a few Kelvin, $\rho(T)$ decreases in all $\mathrm{IrO}_{2} \mathrm{NWs}$, as expected for typical metallic behavior (cf. Supplementary Note 2). However, below $T \sim 20 \mathrm{~K}$, $\rho(T)$ displays a $\sqrt{T}$ increase of the $\rho(T)$ upon lowering $T$ over almost two decades in $T(!)$, until a deviation sets in at $\sim 0.5 \mathrm{~K}$. We performed systematic thermal annealing studies to adjust the oxygen contents in the NWs, which indicate that the anomalous low- $T$ transport properties are driven by the presence of $V_{\mathrm{O}}$ 's (ref. ${ }^{23}$ and Supplementary Note 1). This is exemplified in Fig. 2. The top left inset shows a scanning electron microscopy image of NW A. In the oxygenated NW 3 which should contain a negligible amount of $V_{\mathrm{O}}$ 's, $\rho(T)$ decreases monotonically with decreasing $T$, revealing a residual resistivity, $\rho_{\mathrm{B} 0}$, below $\sim 4 \mathrm{~K}$ (top right inset). In contrast, in NWs $\mathrm{A}, \mathrm{B} 1$ and $\mathrm{B} 2$ which contain large amounts of $V_{\mathrm{O}}$ 's, $\rho(T)$ increases with decreasing $T$, manifesting a robust $\rho \propto \sqrt{T}$ law between $\sim 0.5$ and $\sim 20 \mathrm{~K}$. The slope of NW B2 is smaller than that of NW B1, which indicates a decrease in the number density of oxygen vacancies $\left(n_{V_{O}}\right)$ due to prolonged aging (for about 5 months) in the atmosphere. The data explicitly demonstrate that the $\rho \propto \sqrt{T}$ behavior is independent of $B$ up to at least $9 \mathrm{~T}$. The observed behavior is consistent with the $2 \mathrm{CK}$ effect as indicated by the straight solid lines 


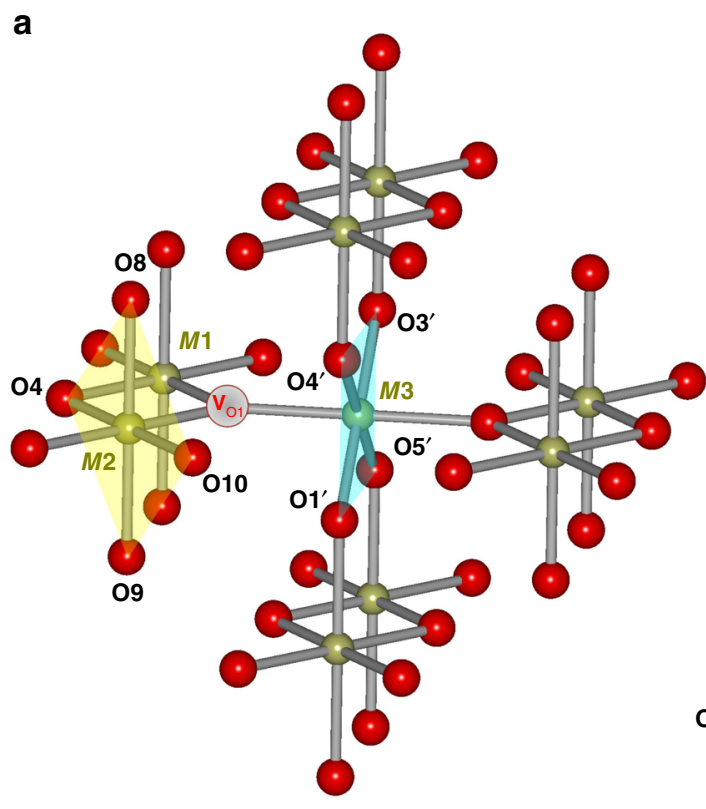

b
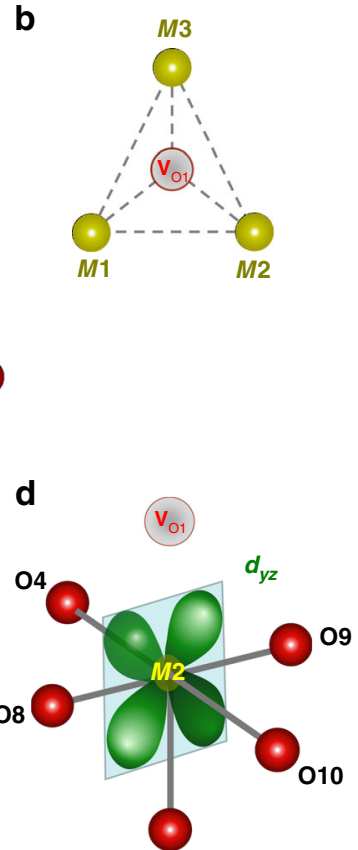

c

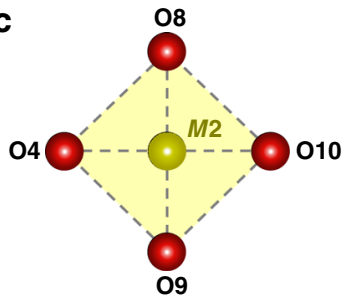

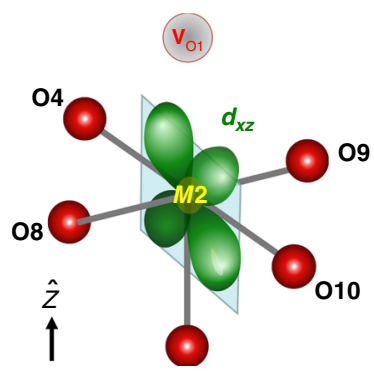

Fig. 1 Atomic arrangement around an oxygen vacancy in $\mathbf{M O}_{\mathbf{2}}$ rutile structure. a Schematics for $\mathrm{MO}_{2}$ in the rutile structure. The olive and red spheres represent transition-metal ions $\mathrm{M}^{4+}$ and oxygen ions $\mathrm{O}^{2-}$, respectively. $V_{01}$ represents an oxygen vacancy. $\mathbf{b}$ The metal ions $M 1, M 2$, and $M 3$ surrounding $V_{\mathrm{O} 1}$ form an isosceles triangle. c The four oxygen ions surrounding $M 2$, labeled $\mathrm{O} 4, \mathrm{O}$, O10, and O8, form an almost perfect planar square (while O1', O5', O3', and O4' only form a rectangle, cf. Supplementary Note 4 for details). d The $d_{x z}$ and $d_{y z}$ orbitals at M2 next to $V_{\mathrm{O} 1}$, with ẑ perpendicular to the O4, O8, $\mathrm{O} 10$, and $\mathrm{O} 9$ plane, remain essentially degenerate as a result of mirror and $C_{4}$ rotation symmetry around $M 2$. (Due to the non-symmorphic rutile structure, $\hat{z} \not \hat{z}^{\prime}$, where $\hat{z}^{\prime}$ is parallel to the $C_{4}$ axis at M1.).

Table 1 Relevant parameters for $\mathrm{MO}_{2} \mathrm{NWs}$.

\begin{tabular}{|c|c|c|c|c|c|c|c|c|c|}
\hline NW & $d$ & $\rho(300 \mathrm{~K})$ & $\rho_{\mathrm{BO}}$ & $\mathscr{\ell}(10 \mathrm{~K})$ & $D(10 K)$ & $\rho_{\text {ко }}$ & $\mathbf{T}_{\mathrm{K}}$ & $n_{v_{o}}$ & $n_{v_{0}} / n_{0}(\%)$ \\
\hline $\mathrm{IrO}_{2} \mathrm{~A}$ & 130 & 147 & 109 & 2.5 & 4.2 & $(0.65)$ & $(20)$ & $\sim 1.9 \times 10^{25}$ & $\sim 0.031$ \\
\hline $\mathrm{IrO}_{2} \mathrm{B1}$ & 190 & 104 & 73.9 & 3.7 & 6.2 & $(0.72)$ & $(20)$ & $\sim 2.2 \times 10^{25}$ & $\sim 0.036$ \\
\hline $\mathrm{IrO}_{2} \mathrm{~B} 2$ & 190 & 106 & 75.0 & 3.6 & 6.0 & $(0.45)$ & $(20)$ & $\sim 1.4 \times 10^{25}$ & $\sim 0.023$ \\
\hline $\mathrm{RuO}_{2} \mathrm{~A}$ & 53 & 193 & 122 & 2.2 & 4.0 & 0.94 & 3.0 & $\sim 1.5 \times 10^{25}$ & $\sim 0.025$ \\
\hline $\mathrm{RuO}_{2} \mathrm{~B}$ & 67 & 163 & 120 & 2.3 & 4.2 & 14 & 12 & $\sim 2.3 \times 10^{26}$ & $\sim 0.38$ \\
\hline $\mathrm{RuO}_{2} \mathrm{C}$ & 54 & 589 & 434 & 0.63 & 1.2 & 17 & 69 & $\sim 2.7 \times 10^{26}$ & $\sim 0.44$ \\
\hline $\mathrm{RuO}_{2} \mathrm{D}$ & 120 & 245 & 160 & 1.7 & 3.1 & 7.0 & 80 & $\sim 1.1 \times 10^{26}$ & $\sim 0.18$ \\
\hline $\mathrm{RuO}_{2} \mathrm{E}$ & 47 & 761 & 587 & 0.47 & 0.9 & 30 & 7.0 & $\sim 4.8 \times 10^{26}$ & $\sim 0.79$ \\
\hline
\end{tabular}

which are linear fits to the $2 \mathrm{CK}$ effect calculated within the dynamical large- $N$ method (cf. Supplementary Note 5 ), with $n_{V_{\mathrm{O}}}$ as an adjustable parameter (see Table 1 for the extracted values and Supplementary Notes 5 and 6 for the extraction method).

Ruling out the 3D electron-electron interaction (EEI) effect. To complicate matters, the EEI effect in 3D weakly disordered metals generically leads to a $\sqrt{T}$ term in $\rho(T)$ at low $T$ (refs. ${ }^{24,25}$ ). Unambiguously establishing that $\rho(T) \sim \sqrt{T}$ indeed originates from $2 \mathrm{CK}$ physics thus requires a proper analysis of the EEI effect of the charge carriers. For example, for the NW B1 with $\rho_{\mathrm{B} 0}=74 \mu \Omega$ $\mathrm{cm}$ and the electron diffusion constant $D \simeq 6.2 \mathrm{~cm}^{2} \mathrm{~s}^{-1}$, the 3D EEI effect would predict a largest possible resistance increase of $\Delta \rho / \rho \simeq$ $2.8 \times 10^{-4}$ as $T$ is cooled from 20 to $1 \mathrm{~K}$. Experimentally, we have observed a much larger resistance increase of $5.1 \times 10^{-3}$. Furthermore, the 3D EEI effect would predict similar values for the magnitude of the low- $T$ resistivity increase in NWs B1 and B2 to within $\approx 3 \%$, due to their $\rho_{\mathrm{B} 0}$ values differing by $\approx 1 \%$ (Table 1 ). This is definitely incompatible with our observation of a $\approx 50 \%$ difference. In addition, we find a deviation from the $\sqrt{T}$ behavior at $\sim 0.5 \mathrm{~K}$. If the $\sqrt{T}$ anomaly were caused by the EEI effect, no such deviation should occur (see Supplementary Note 3 for an in-depth analysis of the EEI effect and its 3D dimensionality in our $\mathrm{MO}_{2} \mathrm{NWs}$ ).

$V_{\mathrm{O}}$-driven orbital Kondo scattering in $\mathrm{MO}_{2}$. For $\mathrm{IrO}_{2}$ the valency of the transition-metal ion $M$ is close to the nominal valence of $+\mathrm{IV}$ in $\mathrm{MO}_{2}$ (ref. $\left.{ }^{26}\right)$. Each $V_{\mathrm{O}}$ generates two defect electrons due to charge neutrality. To minimize Coulomb interaction, the defect electrons will tend to localize at different $M$ ions in the vicinity of the $V_{\mathrm{O}}$. In $\mathrm{IrO}_{2}$ this results in a nonmagnetic $5 d^{6}$ ground state configuration of the Ir ions. For the electron 


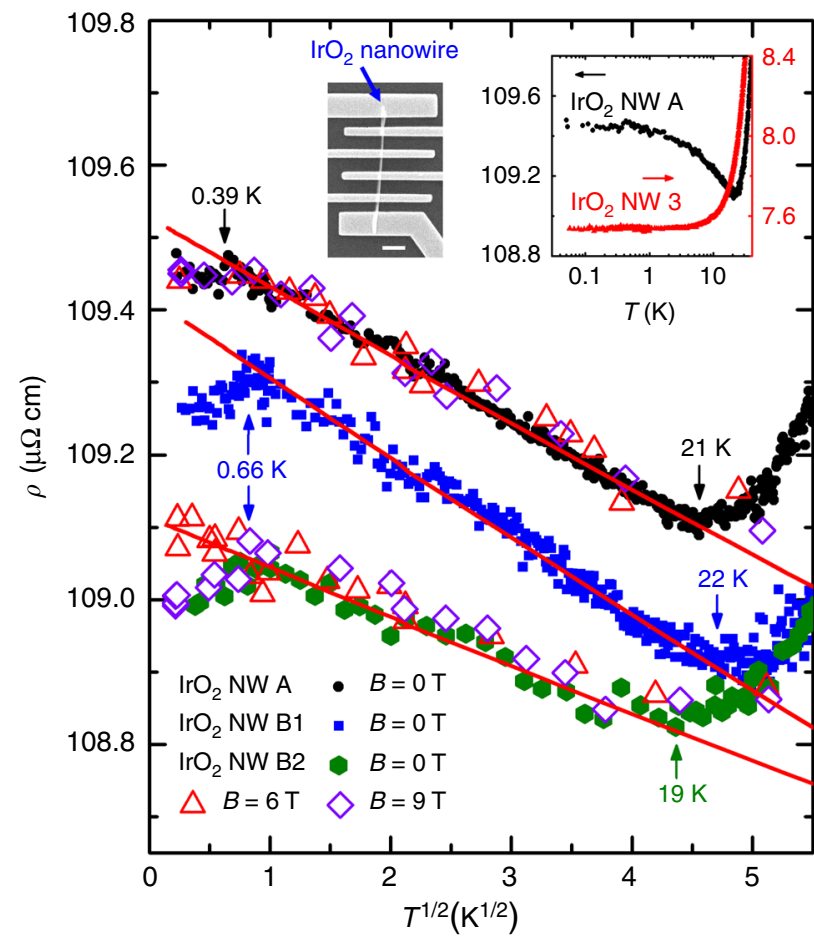

Fig. 2 Orbital 2CK resistivity of $\mathrm{IrO}_{\mathbf{2}} \mathbf{N W s} . \rho$ versus $\sqrt{T}$ for $\operatorname{IrO}_{2} \mathrm{NWs} A$, $B 1$ and $B 2$ in magnetic fields $B=0,6$, and $9 \mathrm{~T}$, as indicated. For clarity, the data of NWs B1 and B2 are shifted by 34.7 and $33.6 \mu \Omega \mathrm{cm}$, respectively. $A$ $\rho \propto \sqrt{T}$ law, which is $B$ independent, is observed between $\sim 0.5$ and $\sim 20 \mathrm{~K}$ in all three NWs. The straight solid lines are linear fits to the $2 \mathrm{CK}$ resistivities calculated by the dynamical large- $N$ method (see text). Top left inset: a scanning electron microscopy image of NW A. The scale bar is $1 \mu \mathrm{m}$. Top right inset: Low-T $\rho(T)$ curves of NW A and a reference, oxygenated NW 3 (diameter $d=330 \mathrm{~nm}, \rho(300 \mathrm{~K})=124 \mu \Omega \mathrm{cm}$ ).

localizing on ion $M 2$ or $M 1$ (Fig. 1a), the symmetry of the effective potential implies the almost perfect degeneracy of the orbitals $d_{x z}$ and $d_{y z}$ as defined in Fig. 1d. It is this orbital degeneracy that drives the orbital 2CK effect in $\mathrm{IrO}_{2}$ where the $d_{x z}$ and $d_{y z}$ form a local pseudospin basis, while the spin-degenerate conduction electrons act as two independent screening channels. Group theoretical arguments ensure that the exchange scattering processes between conduction electrons and pseudospin degree of freedom have a form compatible with the Kondo interaction ${ }^{27}$ (cf. Supplementary Note 5). Deviations from perfect symmetry which act as a pseudo-magnetic field are expected to become visible at lowest $T$. This explains the deviations from the $\sqrt{T}$ behavior observed below $\sim 0.5 \mathrm{~K}$ in Fig. 2. If the two defect electrons localize at sites $M 1$ and $M 2$, a two-impurity problem might be expected which could lead to inter-site orbital order between the two defect electrons ${ }^{28}$. The non-symmorphic rutile structure, however, ensures that the $C_{4}$ rotation axes centered at the sites $M 1$ and $M 2$ are not parallel. This together with the local nature of the decomposition provided in Supplementary Eq. (3) (see Supplementary Note 5) favor local orbital Kondo screening in line with our observation. These conclusions are further corroborated by demonstrating tunability of the orbital $2 \mathrm{CK}$ effect to its $1 \mathrm{CK}$ counterpart.

Experimental signatures of orbital $1 \mathrm{CK}$ effect in $\mathrm{RuO}_{2} \mathrm{NWs}$. $\mathrm{RuO}_{2}$ is also a DNL metal with the same non-symmorphic symmetry group as $\mathrm{IrO}_{2}$ but weaker SOC. In contrast to $\mathrm{IrO}_{2}$, it lacks time-reversal symmetry ${ }^{19,29}$. Based on the analysis for $\mathrm{IrO}_{2}$, we expect that $V_{\mathrm{O}}$ 's in $\mathrm{RuO}_{2}$ will drive an orbital 1CK effect. This is indeed borne out by our transport data on $\mathrm{RuO}_{2}$ NWs. Fig. 3a shows the $T$ dependence of the time-averaged Kondo resistivity $\left\langle\rho_{\mathrm{K}}\right\rangle$ for NW C, where $\rho_{\mathrm{K}}(T)=\rho(T)-\rho_{\mathrm{B} 0}$, and $\langle\ldots\rangle$ denotes averaging. $\left(\mathrm{RuO}_{2} \mathrm{NWs}\right.$ often demonstrate temporal $\rho$ fluctuations. Details can be found in Supplementary Note 2.) At low $T$, $\left\langle\rho_{\mathrm{K}}\right\rangle$ follows the $1 \mathrm{CK}$ form ${ }^{30}$. The inset demonstrates the recovery of a Fermi-liquid ground state with its characteristic $\left\langle\rho_{\mathrm{K}}\right\rangle \propto T^{2}$ behavior below $\sim 12 \mathrm{~K}$ and unambiguously rules out the $3 \mathrm{D}$ EEI effect. Fig. 3b shows $\rho(T)$ of NW E in $B=0$ and 4 T. For clarity, the $B=0$ data (black symbols) are averaged over time, while the $B=4 \mathrm{~T}$ data (red symbols) are non-averaged to demonstrate the temporal fluctuations of the low- $T \rho(T)$ (ref. ${ }^{31}$ ). Note that, apart from the aforementioned much smaller resistance increase as would be predicted by the 3D EEI effect compared with the experimental results in Fig. $3 a, b$, no $\sqrt{T}$ dependence is detected here. In fact, the low- $T$ resistivity anomalies conform very well to the $1 \mathrm{CK}$ scaling form for three decades in $T / T_{\mathrm{K}}$ (Fig. 4a). Thus, the $3 \mathrm{D}$ EEI effect can be safely ruled out as the root of the observed low- $T$ resistivity anomalies in $\mathrm{RuO}_{2}$ NWs.

As a further demonstration of the $B$-field independence, we present in Fig. $3 \mathrm{c} \rho(T)$ data for NW A in magnetic fields of strength $B=0,3$, and $5 \mathrm{~T}$. With $T_{\mathrm{K}}^{\mathrm{A}}=3 \mathrm{~K}, \mathrm{NW}$ A has the lowest $T_{\mathrm{K}}$ among NWs A-E (Table 1). The data between $50 \mathrm{mK}$ and $10 \mathrm{~K}$, corresponding to $T / T_{\mathrm{K}}=0.017-3.3$, can be well described by the $1 \mathrm{CK}$ function (solid curve). The dash-dotted curves depict the magnetoresistance predicted by the spin- $\frac{1}{2}$ Kondo impurity model ${ }^{30}$ with $g \mu_{\mathrm{B}} B / k_{\mathrm{B}} T_{\mathrm{K}}=1.0,2.0$, and 4.1 , as indicated, where $k_{\mathrm{B}}$ is the Boltzmann constant. Our experimental data clearly demonstrate $B$ independence, ruling out a magnetic origin of this phenomenon.

We remark on the relation between the residual resistivity $\rho_{\mathrm{B} 0}$ and the concentration of orbital Kondo scatterers $n_{V_{\mathrm{O}}}$ extracted from $\rho_{\mathrm{K} 0}$, the Kondo contribution to the $\rho(T \rightarrow 0)$ (see Supplementary Note 6), for $\mathrm{RuO}_{2}$ NWs. With the exception of $\mathrm{NW} \mathrm{B}$, our data indicate an approximately linear relation between $n_{V_{\mathrm{O}}}$ and $\rho_{\mathrm{B} 0}$ (Table 1 and Supplementary Fig. 3 ). It is not unexpected that the approximately linear relation between $\rho_{\mathrm{B} 0}$ and $n_{V_{\mathrm{O}}}$ holds for larger impurity concentrations, corresponding to larger values of $\rho_{\mathrm{B} 0}$ as all defects, screened dynamic and static defects, contribute to $\rho_{\mathrm{B} 0}$. This relation strongly demonstrates that the low- $T$ resistivity anomalies are indeed due to $V_{\mathrm{O}}$-driven orbital Kondo effect. (We focus on $\mathrm{RuO}_{2} \mathrm{NWs}$ because of the larger number of samples with a larger variation of $\rho_{\mathrm{B} 0}$ values compared with $\mathrm{IrO}_{2} \mathrm{NWs}$ ).

Comparison of $2 \mathrm{CK}$ and $1 \mathrm{CK} \boldsymbol{\rho}(\mathrm{T})$ curves. Figure $4 \mathrm{a}$ demonstrates that $\left\langle\rho_{\mathrm{K}}\right\rangle / \rho_{\mathrm{K} 0}$ for $\mathrm{RuO}_{2}$ NWs follow the universal 1CK scaling over three decades in $T / T_{\mathrm{K}}$ while $T_{\mathrm{K}}$ ranges from 3 to 80 $\mathrm{K}$ ! To further substantiate the subtle but distinct differences between the $\sqrt{T}$ dependence of the $2 \mathrm{CK}$ behavior in $\mathrm{IrO}_{2} \mathrm{NWs}$ from the 1CK scaling form, we plot $\left\langle\rho_{\mathrm{K}}\right\rangle / \rho_{\mathrm{K} 0}$ as a function of $\sqrt{T / T_{\mathrm{K}}}$ for $\mathrm{IrO}_{2}$ NWs $\mathrm{A}$ and $\mathrm{B} 1$, together with $\mathrm{RuO}_{2}$ NWs B-E and the $1 \mathrm{CK}$ function, in Fig. $4 \mathrm{~b}$, c, respectively. (The value for $\rho_{\mathrm{K} 0}$ was identified with the maximum values of the measured $\rho_{\mathrm{K}}(T)$ anomalies.) Fig. $4 \mathrm{~d}$ illustrates that a dilute system of $2 \mathrm{CK}$ scattering centers immersed in a metallic host indeed displays a $\sqrt{T}$ term in its low- $T \rho(T)$. This $\sqrt{T}$ power-law behavior is determined by the leading irrelevant operator near the $2 \mathrm{CK}$ fixed point $^{32}$ and captured by the dynamical large- $N$ method ${ }^{33-35}$.

\section{Discussion}

Despite the ubiquitous appearance of magnetic Kondo scattering in real quantum materials ${ }^{36}$, no convincing demonstration of the orbital Kondo effect ${ }^{37}$ or the $2 \mathrm{CK}$ effect ${ }^{22,38}$ exists. Many claims rest on a model of two-level systems immersed in a metallic host as a 

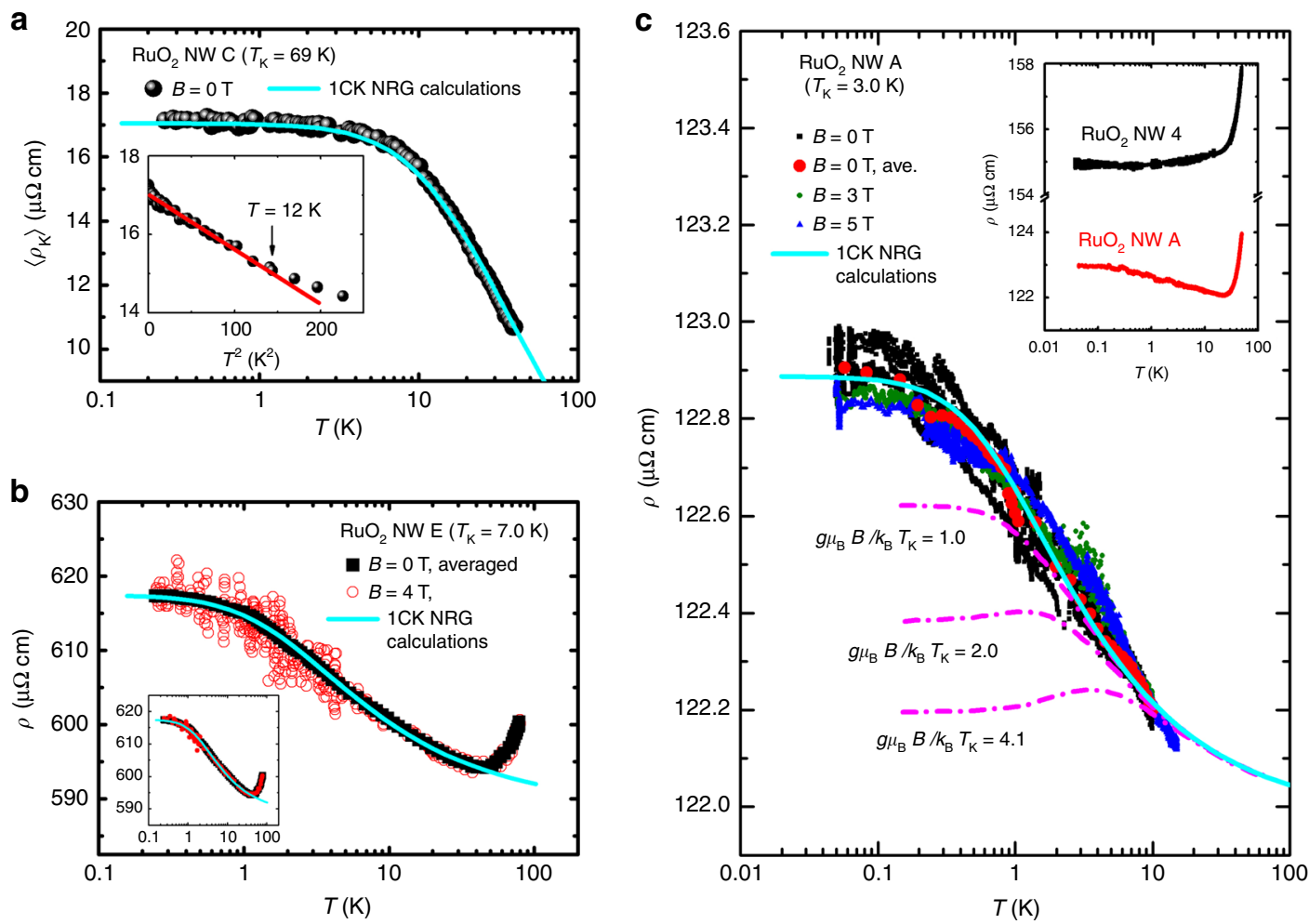

Fig. 3 Orbital 1CK resistivity of $\mathbf{R u O}_{\mathbf{2}} \mathbf{N W s}$. a Time-averaged Kondo resistivity $\left\langle\rho_{\mathrm{K}}\right\rangle$ versus $\log T$ for NW C. The straight line in the inset, which shows a low- $T$ zoom-in, is a guide to the eye. $\mathbf{b} \rho$ versus $\log T$ in $B=0$ and $4 T$ for NW E. For clarity, the $B=0$ data are time-averaged, while the 4-T data are nonaveraged to demonstrate the temporal resistivity fluctuations at low $T$. The inset shows the time-averaged $B=4 \mathrm{~T}$ data (red symbols), which closely overlap the $B=0$ data. c $\rho$ versus $\log T$ for NW A in $B=0,3$, and $5 \mathrm{~T}$. Occasional resistivity jumps, or random telegraph noise, are observed. The dashdotted curves depict the magnetoresistance predicted by the spin- $\frac{1}{2}$ Kondo impurity model (see text). Note that the experimental data are independent of $B$. Inset: Low- $T \rho(T)$ curves of NW A and a reference, oxygenated NW $4(d=150 \mathrm{~nm}, \rho(300 \mathrm{~K})=336 \mu \Omega \mathrm{cm})$. In a-c, the solid curve shows the $B=0$ numerical renormalization group result for $1 \mathrm{CK}$ effect ${ }^{59}$.

possible route to $2 \mathrm{CK}$ physics ${ }^{3,4}$. Theoretical arguments have, however, made it clear that this is not a viable route to nonmagnetic Kondo scattering ${ }^{22,38}$. Moreover, the creation of scattering centers in a real quantum material necessarily places the system in the weakly disordered regime where a conductance anomaly, the Altshuler-Aronov correction, occurs whose $T$ dependence can be mistaken for a $2 \mathrm{CK}$ signature, see, e.g., refs. ${ }^{39-42}$. Dilution studies on common Kondo lattice systems ${ }^{43,44}$, on the other hand, typically create disorder distributions of Kondo temperatures that may result in a behavior of observables, which can easily be mistaken for that of a generic non-Fermi liquid ${ }^{45}$.

We have shown that the low- $T$ resistivity anomaly in the transition-metal rutile $\mathrm{IrO}_{2}$ is caused by $V_{\mathrm{O}}$ 's, demonstrating key signatures of an orbital $2 \mathrm{CK}$ effect and ruling out alternative explanations due to, e.g., the EEI effect. The most convincing argument in favor of $2 \mathrm{CK}$ physics would be the demonstration of direct tunability of $2 \mathrm{CK}$ physics to $1 \mathrm{CK}$ physics upon breaking the channel degeneracy. This is difficult, as the channel degeneracy is protected by time-reversal symmetry. A perhaps less direct, yet complementary, demonstration of this tunability is provided by our results for $\mathrm{RuO}_{2} \mathrm{NWs}$ which develop an orbital 1CK effect. In $\mathrm{RuO}_{2}$, the antiferromagnetic order breaks the channel degeneracy. Our analysis also indicates that the underlying symmetries which support the existence of DNLs in the Brillouin zones of both transition-metal rutiles also aid the formation of orbital 2CK and 1CK physics.

Materials condensing in the rutile structure type and its derivatives form an abundant and important class that has helped shaping our understanding of correlated matter. The metalinsulator transition in $\mathrm{VO}_{2}$, e.g., has been known for 60 years ${ }^{46}$, yet its dynamics is still not fully understood ${ }^{47}$. The demonstration that the non-symmorphic rutile space group supports a $V_{\mathrm{O}^{-}}$driven orbital Kondo effect in $\mathrm{MO}_{2}$ holds promise for the realization of novel states of matter. The potential richness of orbital Kondo physics, e.g., on superconducting pairing, was recently pointed out in ref. ${ }^{37}$ but may be even richer when considering the possibility of its interplay with topological band structures. Specifically, we envision the creation of a $2 \mathrm{CK}$ non-symmorphic superlattice of $V_{\mathrm{O}}$ 's in $\mathrm{IrO}_{2}$ where the 2CK Majorana modes entangle with the band structure-enforced Dirac excitations forming a strongly correlated topological non-Fermi liquid state. Understanding its properties will foster deeper insights into the interplay of topology with strong correlations beyond the usual mean field treatment. The theoretical approach to this nonsymmorphic superlattice is reminiscent of the topologically garnished strong-coupling fixed-point pioneered in the context of Weyl-Kondo semimetals ${ }^{11,48}$, suitably generalized to capture the intermediate coupling physics of the $2 \mathrm{CK}$ effect and its low- $T$ excitations. The fabrication of superlattices of Kondo scattering centers has already been demonstrated ${ }^{49}$ while defect engineering of vacancy networks, including $V_{\mathrm{O}}$ networks is currently explored in a range of materials ${ }^{50,51}$. The specifics of this unique state and its manufacturing are currently being explored.

\section{Methods}

NW growth. $\mathrm{IrO}_{2} \mathrm{NWs}$ were grown by the metal-organic chemical vapor deposition method, using (MeCp)Ir(COD) supplied by Strem Chemicals as the source reagent. Both the precursor reservoir and the transport line were controlled in the temperature range of $100-130^{\circ} \mathrm{C}$ to avoid precursor condensation during the vapor-phase transport. High purity $\mathrm{O}_{2}$, with a flow rate of $100 \mathrm{sccm}$, was used as the carrier gas and reactive gas. During the deposition, the substrate temperature 

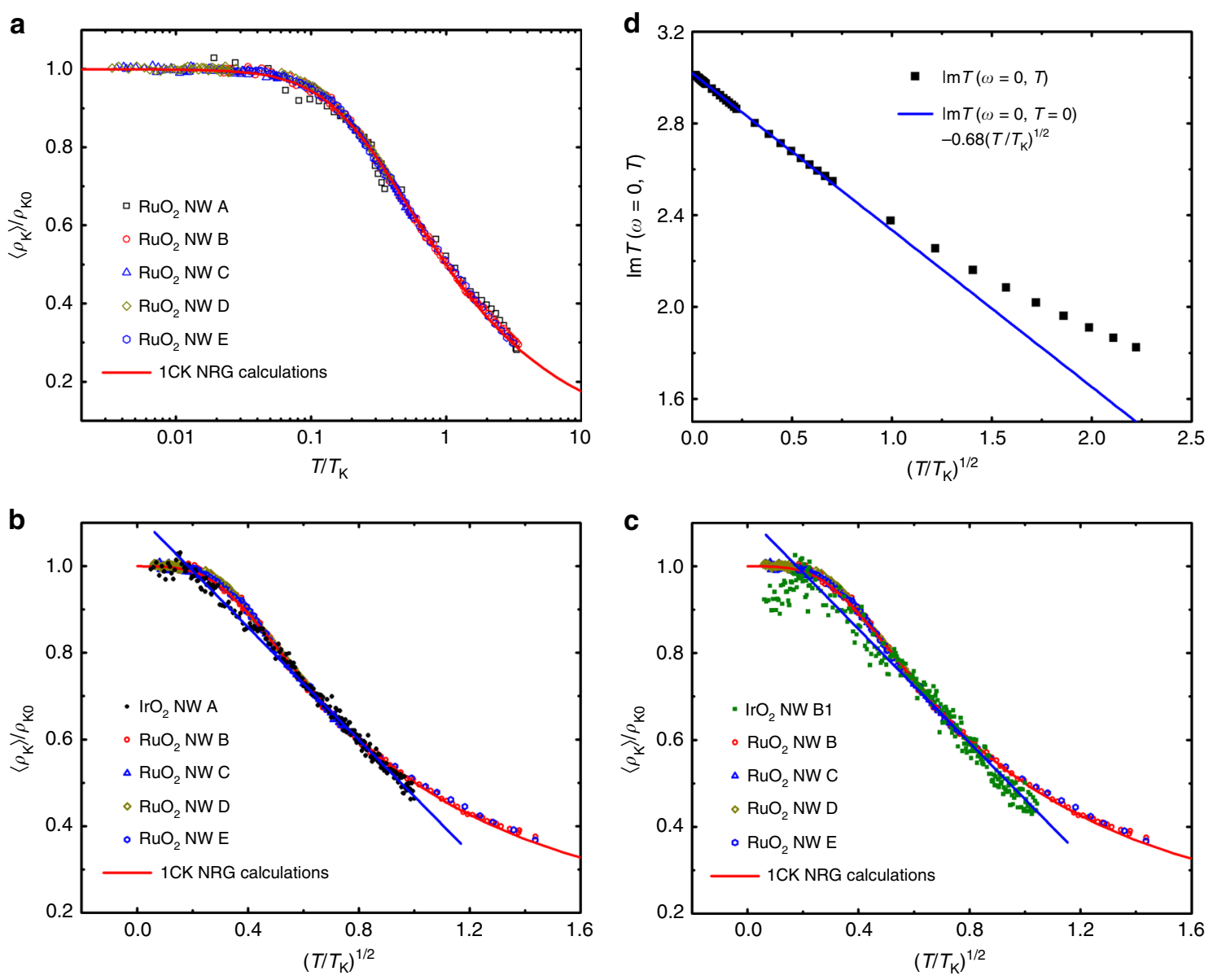

Fig. 4 Comparison of $\mathbf{2 C K}$ and $\mathbf{1 C K}$ resistivities. a Normalized Kondo resistivity $\left\langle\rho_{\mathrm{K}}\right\rangle / \rho_{\mathrm{Ko}}$ versus $T / T_{\mathrm{K}}$ for RuO $2 \mathrm{NWs} A-E$ manifests the $1 C K$ scaling form (solid curve) for over three decades of reduced temperature. $\mathbf{b}\left\langle\rho_{\mathrm{K}}\right\rangle / \rho_{\mathrm{KO}}$ versus $\sqrt{T / T_{\mathrm{K}}}$ for $\operatorname{IrO}_{2} \mathrm{NW} \mathrm{A}$ and $\mathrm{RuO}_{2} \mathrm{NWs} \mathrm{B}-\mathrm{E}$. The data of $\operatorname{IrO} \mathrm{O}_{2} \mathrm{NW} \mathrm{A}$ obeys a $\sqrt{T}$ law between 0.39 and $21 \mathrm{~K}$. For clarity, the experimental data points for $\mathrm{RuO}_{2} \mathrm{NWs}$ are plotted with small open symbols. c $\left\langle\rho_{\mathrm{K}}\right\rangle / \rho_{\mathrm{KO}}$ of $\operatorname{lrO} \mathrm{O}_{2} \mathrm{NW} \mathrm{B} 1$ obeys a $\sqrt{T / T_{K}}$ law between 0.66 and $22 \mathrm{~K}$, distinctively deviating from the $1 C \mathrm{~K}$ function. $\mathbf{d}$ Results for the resistivity of a diluted system of $2 \mathrm{CK}$ impurities in a metallic host evaluated using a dynamical large- $N$ limit (black symbols), which follows a $\sqrt{T / T_{\mathrm{K}}}$ law at low $T$ (see text and Supplementary Note 5). The ordinate is plotted in unit of half-bandwidth $W=4 \mathrm{eV}$ (ref. ${ }^{60}$ ).

was kept at $\approx 350^{\circ} \mathrm{C}$ and the chamber pressure was held at $\approx 17$ torr to grow $\mathrm{NWs}^{52,53}$. Selected-area electron diffraction patterns ${ }^{52}$ and X-ray diffraction (XRD) patterns ${ }^{54}$ revealed a single-crystalline rutile structure.

$\mathrm{RuO}_{2} \mathrm{NWs}$ were grown by the thermal evaporation method based on the vaporliquid-solid mechanism, with Au nanoparticles as catalyst. A quartz tube was inserted in a furnace. A source material of stoichiometric $\mathrm{RuO}_{2}$ powder (Aldrich, 99.9\%) was placed in the center of the quartz tube and heated to $920-960^{\circ} \mathrm{C}$. During the NW growth, an $\mathrm{O}_{2}$ gas was introduced into the quartz tube and the chamber was maintained at a constant pressure of $\approx 2$ torr. Silicon wafer substrates were loaded at the downstream end of the quartz tube, where the temperature was kept at $450-670{ }^{\circ} \mathrm{C}\left(\right.$ ref. ${ }^{55}$ ). The morphology and lattice structure of the NWs were studied using XRD and high-resolution transmission electron microscopy (HRTEM). The XRD patterns demonstrated a rutile structure ${ }^{55}$, and the HR-TEM images revealed a polycrystalline lattice structure ${ }^{56}$.

Electrical measurements. Submicron $\mathrm{Cr} / \mathrm{Au}(10 / 100 \mathrm{~nm})$ electrodes for 4-probe $\rho(T)$ measurements were fabricated by the standard electron-beam lithography technique. The electrode fabrication was done after the thermal treatment (annealing and/or oxygenation) of each NW was completed. To avoid electron overheating, the condition for equilibrium, $e V_{\mathrm{s}} \ll k_{\mathrm{B}} T$, was assured in all resistance measurements ${ }^{57}$, where $e$ is the electronic charge, and $V_{\mathrm{s}}$ is the applied voltage across the energy relaxation length. The electrical-transport measurements were performed on a BlueFors LD-400 dilution refrigerator equipped with roomtemperature and low-temperature low-pass filters. The electron temperature was calibrated down to $\lesssim 50 \mathrm{mK}$. In several cases $\left(\mathrm{RuO}_{2} \mathrm{NWs} \mathrm{B}-\mathrm{E}\right)$, the measurements were performed on an Oxford Heliox ${ }^{3} \mathrm{He}$ cryostat with a base temperature of $\simeq 250 \mathrm{mK}$. The magnetic fields were supplied by superconducting magnets and applied perpendicular to the NW axis in all cases.

\section{Data availability}

All data collected or analyzed during this study is available in the main text or the Supplementary Information material.

\section{Code availability}

Details on the numerics is available upon request from the authors.

Received: 7 April 2020; Accepted: 18 August 2020;

Published online: 21 September 2020

\section{References}

1. Keimer, B., Kivelson, S. A., Norman, M. R., Uchida, S. \& Zaanen, J. From quantum matter to high-temperature superconductivity in copper oxides. Nature 518, 179-186 (2015).

2. Noziéres, P. \& Blandin, A. Kondo effect in real metals. J. Phys. 41, 193-211 (1980).

3. Vladár, K. \& Zawadowski, A. Theory of the interaction between electrons and the two-level system in amorphous metals. I. Noncommutative model Hamiltonian and scaling of first order. Phys. Rev. B 28, 1564-1581 (1983).

4. Cox, D. L. \& Zawadowski, A. Exotic Kondo effects in metals: magnetic ions in a crystalline electric field and tunnelling centres. Adv. Phys. 47, 599-942 (1998).

5. Potok, R. M., Rau, I. G., Shtrikman, H., Oreg, Y. \& Goldhaber-Gordon, D. Observation of the two-channel Kondo effect. Nature 446, 167-171 (2007). 
6. Keller, A. J. et al. Universal Fermi liquid crossover and quantum criticality in a mesoscopic system. Nature 526, 237 (2015).

7. Iftikhar, Z. et al. Two-channel Kondo effect and renormalization flow with macroscopic quantum charge states. Nature 526, 233 (2015).

8. Iftikhar, Z. et al. Quantum criticality and super-ballistic transport in a charge Kondo circuit. Science 360, 1315 (2018).

9. Burkov, A. A. Topological semimetals. Nat. Mater. 15, 1145-1148 (2016)

10. Yang, S.-Y. et al. Symmetry demanded topological nodal-line materials. Adv. Phys.: X 3, 1414631 (2018)

11. Dzsaber, S. et al. Kondo insulator to semimetal transformation tuned by spinorbit coupling. Phys. Rev. Lett. 118, 246601 (2017).

12. Ourmazd, A. \& Spence, J. C. H. Detection of oxygen ordering in superconducting cuprates. Nature 329, 425-427 (1987).

13. Lin, C. \& Demkov, A. A. Electron correlation in oxygen vacancy in $\mathrm{SrTiO}_{3}$ Phys. Rev. Lett. 111, 217601 (2013).

14. Lin, C., Shin, D. \& Demkova, A. A. Localized states induced by an oxygen vacancy in rutile $\mathrm{TiO}_{2}$. J. Appl. Phys. 117, 225703 (2015).

15. Lechermann, F., Heckel, W., Kristanovski, O. \& Müller, S. Oxygen-vacancy driven electron localization and itinerancy in rutile-based $\mathrm{TiO}_{2}$. Phys. Rev. B 95, 195159 (2017).

16. Jovic, V. et al. Dirac nodal lines and flat-band surface state in the functional oxide $\mathrm{RuO}_{2}$. Phys. Rev. B 98, 241101 (2018).

17. Nelson, J. N. et al. Dirac nodal lines protected against spin-orbit interaction in $\mathrm{IrO}_{2}$. Phys. Rev. Mater. 3, 064205 (2019).

18. Zhao, Y. X. \& Schnyder, A. P. Nonsymmorphic symmetry-required band crossings in topological semimetals. Phys. Rev. B 94, 195109 (2016).

19. Sun, Y., Zhang, Y., Liu, C.-X., Felser, C. \& Yan, B. Dirac nodal lines and induced spin Hall effect in metallic rutile oxides. Phys. Rev. B 95, 235104 (2017).

20. Coleman, P., Ioffe, L. B. \& Tsvelik, A. M. Simple formulation of the twochannel Kondo model. Phys. Rev. B 52, 6611-6627 (1995).

21. Moustakas, A. L. \& Fisher, D. S. Two-channel Kondo physics from tunneling impurities with triangular symmetry. Phys. Rev. B 55, 6832-6846 (1997).

22. Aleiner, I. \& Controzzi, D. Nonexistence of a strong coupling two-channel Kondo fixed point for microscopic models of tunneling centers. Phys. Rev. B 66, 045107 (2002).

23. Yeh, S.-S., Gao, K. H., Wu, T.-L., Su, T.-K. \& Lin, J.-J. Activation energy distribution of dynamical structural defects in $\mathrm{RuO}_{2}$ films. Phys. Rev. Appl. 10, 034004 (2018).

24. Altshuler, B. L. \& Aronov, A. G. Electron-electron Interaction in Disordered Conductors, book section 1 (North-Holland Physics Publishing, Amsterdam, The Netherlands, 1985).

25. Lee, P. A. \& Ramakrishnan, T. V. Disordered electronic systems. Rev. Mod. Phys. 57, 287-337 (1985).

26. Ping, Y., Galli, G. \& Goddard, W. A. Electronic structure of $\mathrm{IrO}_{2}$ : the role of the metal d orbitals. J. Phys. Chem. C. 119, 11570-11577 (2015).

27. Cox, D. L. Quadrupolar Kondo effect in uranium heavy-electron materials? Phys. Rev. Lett. 59, 1240-1243 (1987).

28. Mitchell, A. K., Sela, E. \& Logan, D. E. Two-channel Kondo physics in twoimpurity Kondo models. Phys. Rev. Lett. 108, 086405 (2012).

29. $\mathrm{Zhu}, \mathrm{Z}$. H. et al. Anomalous antiferromagnetism in metallic $\mathrm{RuO}_{2}$ determined by resonant X-ray scattering. Phys. Rev. Lett. 122, 017202 (2019).

30. Costi, T. A. Kondo effect in a magnetic field and the magnetoresistivity of Kondo alloys. Phys. Rev. Lett. 85, 1504-1507 (2000).

31. Lien, A.-S., Wang, L. Y., Chu, C. S. \& Lin, J.-J. Temporal universal conductance fluctuations in $\mathrm{RuO}_{2}$ nanowires due to mobile defects. Phys. Rev. B 84, 155432 (2011).

32. Affleck, I. \& Ludwig, A. W. Exact conformal-field-theory results on the multichannel Kondo effect: single-fermion Green's function, self-energy, and resistivity. Phys. Rev. B 48, 7297-7321 (1993).

33. Parcollet, O. \& Georges, A. Overscreened multichannel SU(N) Kondo model: large-N solution and conformal field theory. Phys. Rev. B 58, 3794-3813 (1998).

34. Cox, D. \& Ruckenstein, A. Spin-flavor separation and non-Fermi-liquid behavior in the multichannel Kondo problem: a large-N approach. Phys. Rev. Lett. 71, 1613-1616 (1993).

35. Zamani, F., Chowdhury, T., Ribeiro, P., Ingersent, K. \& Kirchner, S. Quantum criticality in the two-channel pseudogap Anderson model: a test of the noncrossing approximation. Phys. Status Solidi B 250, 547-552 (2013).

36. Hewson, A. C. The Kondo Problem to Heavy Fermions. (Cambridge University Press, Cambridge, 1993).

37. Kuramoto, Y. Composite electronic orders induced by orbital Kondo effect. Sci. Bull. 61, 1563 (2016).

38. Moustakas, A. \& Fisher, D. Prospects for non-Fermi-liquid behavior of a twolevel impurity in a metal. Phys. Rev. B 53, 4300-4315 (1996).

39. Zhu, L. J., Nie, S. H., Xiong, P., Schlottmann, P. \& Zhao, J. H. Orbital twochannel Kondo effect in epitaxial ferromagnetic $\mathrm{L}_{0}-\mathrm{MnAl}$ films. Nat. Commun. 7, 10817 (2016).
40. Zhu, L. J. \& Zhao, J. H. Anomalous resistivity upturn in epitaxial $\mathrm{L}_{1}-\mathrm{Co}_{2}$ MnAl films. Sci. Rep. 7, 42931 (2017).

41. Cichorek, T. et al. Two-channel Kondo physics due to As vacancies in the layered compound $\mathrm{ZrAs}_{1.58} \mathrm{Se}_{0.39}$. Phys. Rev. Lett. 117, 106601 (2016).

42. Gnida, D. Comment on two-channel Kondo physics due to As vacancies in the layered compound $\mathrm{ZrAs}_{1.58} \mathrm{Se}_{0.39}$. Phys. Rev. Lett. 118, 259701 (2017).

43. Nicklas, $M$. et al. Charge-doping-driven evolution of magnetism and nonFermi-liquid behavior in the filled skutterudite $\mathrm{CePt}_{4} \mathrm{Ge}_{12-x} \mathrm{Sb}_{x}$. Phys. Rev. Lett. 109, 236405 (2012).

44. Yamane, Y. et al. Single-site non-Fermi-liquid behaviors in a diluted $4 f^{2}$ system $\mathrm{Y}_{1-x} \mathrm{Pr}_{x} \mathrm{Ir}_{2} \mathrm{Zn}_{20}$. Phys. Rev. Lett. 121, 077206 (2018).

45. Miranda, E., Dobrosavljević, V. \& Kotliar, G. Disorder-driven non-Fermiliquid behavior in Kondo alloys. Phys. Rev. Lett. 78, 290-293 (1997).

46. Morin, F. J. Oxides which show a metal-to-insulator transition at the Néel temperature. Phys. Rev. Lett. 3, 34-36 (1959).

47. Wall, S. et al. Ultrafast disordering of vanadium dimers in photoexcited $\mathrm{VO}_{2}$. Science 362, 572-576 (2018).

48. Lai, H.-H., Grefe, S. E., Paschen, S. \& Si, Q. Weyl-Kondo semimetal in heavyfermion systems. PNAS 115, 93-97 (2018).

49. Goh, S. K. et al. Anomalous upper critical field in $\mathrm{CeCoIn}_{5} / \mathrm{YbCoIn}_{5}$ superlattices with a Rashba-type heavy fermion interface. Phys. Rev. Lett. 109, 157006 (2012)

50. Simonov, A. et al. Hidden diversity of vacancy networks in Prussian blue analogues. Nature 578, 256-260 (2020).

51. Lai, F. et al. Oxygen vacancy engineering in spinel-structured nanosheet wrapped hollow polyhedra for electrochemical nitrogen fixation under ambient conditions. J. Mater. Chem. A 8, 1652-1659 (2020).

52. Chen, R.-S. \& Huang, Y.-S. Field emission from vertically aligned conductive $\mathrm{IrO}_{2}$ nanorods. Appl. Phys. Lett. 84, 1552 (2004).

53. Lin, $\mathrm{Y}$. H. et al. Electrical transport studies of individual $\mathrm{IrO}_{2}$ nanorods and their nanorod contacts. Nanotechnology 19, 045711 (2008).

54. Chen, R.-S. et al. Growth and characterization of vertically aligned selfassembled $\mathrm{IrO}_{2}$ nanotubes on oxide substrates. J. Cryst. Growth 271, 105-112 (2004).

55. Liu, Y.-L. et al. Growth of single-crystalline $\mathrm{RuO}_{2}$ nanowires with one- and two-nanocontact electrical characterizations. Appl. Phys. Lett. 90, 013105 (2007).

56. Yeh, S.-S., Chang, W.-Y. \& Lin, J.-J. Probing nanocrystalline grain dynamics in nanodevices. Sci. Adv. 3, e1700135 (2017).

57. Huang, S. M., Lee, T. C., Akimoto, H., Kono, K. \& Lin, J. J. Observation of strong electron dephasing in highly disordered $\mathrm{Cu}_{93} \mathrm{Ge}_{4} \mathrm{Au}_{3}$ thin films. Phys. Rev. Lett. 99, 046601 (2007).

58. Momma, K. \& Izumi, F. VESTA3 for three-dimensional visualization of crystal, volumetric and morphology data. J. Appl. Crystallogr. 44, 1272-1276 (2011).

59. Costi, T. A., Hewson, A. C. \& Zlatic, V. Transport coefficients of the Anderson model via the numerical renormalization group. Matter 6, 2519-2558 (1994).

60. de Almeida, J. S. \& Ahuja, R. Electronic and optical properties of $\mathrm{RuO}_{2}$ and $\mathrm{IrO}_{2}$. Phys. Rev. B 73, 165102 (2006).

\section{Acknowledgements}

The authors are grateful to F. R. Chen, J. J. Kai, and the late Y. S. Huang for growing $\mathrm{RuO}_{2}$ and $\mathrm{IrO}_{2} \mathrm{NWs}$, S. P. Chiu for experimental assistance, T. A. Costi for providing the 1CK scaling curve from NRG calculations, and Q. Si, A. M. Chang, C. H. Chung, and S. Wirth for helpful discussions. Figure la was produced with the help of VESTA ${ }^{58}$. This work was supported by Ministry of Science and Technology, Taiwan (grant Nos. MOST 106-2112-M-009-007-MY4, 108-3017-F-009-004, and 108-2811-M-009-500) and the Center for Emergent Functional Matter Science of National Chiao Tung University from The Featured Areas Research Center Program within the framework of the Higher Education Sprout Project by the Ministry of Education (MOE) in Taiwan. F.Z. and J.K. acknowledge financial support by the Deutsche Forschungsgemeinschaft (DFG) through SFB/TR 185 (277625399) and the Cluster of Excellence ML4Q (390534769). Work at Zhejiang University was in part supported by the National Key R\&D Program of the MOST of China, grant No. 2016YFA0300202 and the National Science Foundation of China, grant No. 11774307.

\section{Author contributions}

S.S.Y., S.K., and J.J.L. conceived the experiment. S.S.Y. and A.S.L. carried out electricaltransport measurements. T.K.S., C.C.L., and A.S.L. fabricated 4-probe NW devices with thermal treatments. F.Z. performed dynamical large- $N$ calculations. J.K. provided theoretical support. S.S.Y., S.K., and J.J.L. analyzed and explained the data, and wrote the paper.

\section{Competing Interests}

The authors declare no competing interests. 


\section{Additional information}

Supplementary information is available for this paper at https://doi.org/10.1038/s41467020-18407-7.

Correspondence and requests for materials should be addressed to S.K. or J.-J.L.

Peer review information Nature Communications thanks the anonymous reviewer(s) for their contribution to the peer review of this work. Peer reviewer reports are available.

Reprints and permission information is available at http://www.nature.com/reprints

Publisher's note Springer Nature remains neutral with regard to jurisdictional claims in published maps and institutional affiliations. (c) (i) Open Access This article is licensed under a Creative Commons Attribution 4.0 International License, which permits use, sharing, adaptation, distribution and reproduction in any medium or format, as long as you give appropriate credit to the original author(s) and the source, provide a link to the Creative Commons license, and indicate if changes were made. The images or other third party material in this article are included in the article's Creative Commons license, unless indicated otherwise in a credit line to the material. If material is not included in the article's Creative Commons license and your intended use is not permitted by statutory regulation or exceeds the permitted use, you will need to obtain permission directly from the copyright holder. To view a copy of this license, visit http://creativecommons.org/ licenses/by/4.0/.

(C) The Author(s) 2020 